\title{
El análisis químico en el control de procesos metalúrgicos
}

\author{
A.G. Coedo ${ }^{(*)}, \mathrm{M}^{\mathrm{a}}$. T. Dorado ${ }^{(*)}$ e I. Padilla ${ }^{(*)}$
}

Resumen Se pone de manifiesto el papel del análisis químico en la industria metalúrgica, en cuanto que permite garantizar la calidad de los productos requeridos. Por ejemplo, en la industria siderúrgica permite garantizar las exigencias requeridas por sectores tales como: automoción, aeroespacial, eléctrico, hojalatas para envases de alimentación o medio ambiental. Aunque las tecnologías analíticas continúan avanzando, consiguiéndose mayor rapidez y mejor precisión y exactitud, al tiempo que más bajos niveles de detección, la competitividad de la industria del sector hace que las exigencias crezcan al menos a la misma velocidad. Unas especificaciones más estrictas, un menor contenido de elementos residuales y las presiones económicas prescribiendo procesos más rápidos, conducen a un incremento en las exigencias de la función analítica. Estas connotaciones se ilustran con algunos ejemplos de distintos sectores del mercado, en los cuales se consideran los requerimientos del usuario conjuntamente con las implicaciones analíticas.

Palabras clave: Control de procesos metalúrgicos. Calidad analítica. Rutina analítica.

\section{Analytical control in metallurgical processes}

\begin{abstract}
This paper illustrates the role of analysis in enabling metallurgical industry to meet quality demands. For example, for the steel industry the demands by the automotive, aerospace, power generation, tinplate packaging industries and issue of environment near steel plants. Although chemical analysis technology continues to advance, achieving improved speed, precision and accuracy at lower levels of detection, the competitiveness of manufacturing industry continues to drive property demands at least at the same rate. Narrower specification ranges, lower levels of residual elements and economic pressures prescribe faster process routes, all of which lead to increased demands on the analytical function. These demands are illustrated by examples from several market sectors in which customer issues are considered together with their analytical implications.
\end{abstract}

Keywords: Metallurgical processes control. Analytical quality. Analytical routine.

\section{GENERALIDADES}

Las técnicas y métodos analíticos constituyen la base de la caracterización fisicoquímica de la mayoría de los materiales, caracterización generalmente requerida en todas las áreas de la ciencia y de la tecnología. El deseo y la necesidad de resolver problemas analíticos da lugar a la introducción de nuevas ideas y al posterior desarrollo de las respectivas técnicas.

Sensibilidad y poder de detección, resolución y selectividad, exactitud y precisión, son los retos del presente y del futuro analítico. Por otra parte, temas

${ }^{*}$ ) Centro Nacional de Investigaciones Metalúrgicas, CENIM (CSIC), Avda. de Gregorio del Amo, 8. 28040-Madrid (España). tales como muestreo, preparación y tratamiento de la muestra y relación entre costo e interés del dato obtenido no deben ni pueden ser olvidados.

Se dice que un fenómeno está controlado cuando se puede hacer una predicción (al menos, dentro de unos límites) de cómo se supone que va a evolucionar. El análisis químico puede usarse para controlar procesos tecnológicos, pero, a su vez, los resultados químicos deben ser controlados. No hay que olvidar las crecientes exigencias en cuanto a criterios y requisitos para evaluar la calidad de los resultados analíticos.

Muchas de las modernas técnicas espectroscópicas (FRX, OES, ICP, etc.) con frecuencia originan datos multielementales para una única muestra. Para asegurar la ausencia de errores en los resultados, se requiere la implantación de un sistema de 
control de la calidad de dichos resultados que garantice su Aptitud para el requerimiento (1). Se trata de un principio universalmente aceptado que, aplicado a las medidas analíticas, implica el uso de la metodología más correcta para obtener unos resultados de calidad adecuada. Este concepto se puede definir como "La adecuación de los datos obtenidos por un determinado método analítico, de forma que permitan al usuario de dichos datos la toma de decisiones técnicamente correctas para un propósito predefinido". Este concepto se refiere consiguientemente a la magnitud de la incertidumbre asociada con una medida, en relación con las necesidades del área de aplicación. En determinados campos de análisis, sólo son toleradas incertidumbres relativas muy pequeñas. Así, en un resultado analítico requerido para negociar un precio de una chatarra de metales preciosos, la exactitud que se precisa es muy alta; en contraste, en prospecciones geológicas para metales preciosos, donde el propósito es esencialmente distinguir localizaciones de minerales que contengan bajas (pero interesantes para su explotación) concentraciones de metales preciosos, puede aceptarse una incertidumbre relativa de hasta un $20 \%$. En este último caso, unos resultados con una mayor exactitud pueden ser no sólo innecesarios, sino prohibitivamente costosos.

Como conclusión, se puede decir que la Aptitud para el requerimiento implica una exactitud suficiente, pero sólo la estrictamente necesaria; pudiendo restringirse requerimientos puramente científicos por consideraciones meramente económicas. Normalmente, cuando un material va a ser analizado, el proceso empieza por realizar un muestreo, y a partir de él la toma de muestra para análisis. El resultado final viene, pues, acompañado por dos tipos de error:

\section{Resultado obtenido $=$ resultado verdadero \pm \pm (error muestreo + error analítico)}

De donde se deduce una incertidumbre del resultado, expresada como varianza, dada por la suma de las varianzas del muestreo y del análisis $\left(v_{\mathrm{m}}+v_{\mathrm{a}}\right)$; de ahí, la importancia de conocer la relación óptima entre los valores de dichas varianzas en el contexto de las necesidades del usuario final. Esta relación óptima depende, en parte, de los costos relativos del muestreo y del análisis; sin olvidar el costo de la mejora en la precisión. Suponiendo que el costo del análisis de una muestra por un determinado método, caracterizado con una desviación estándar de 1 , es $X$, el costo de un resultado con una mayor precisión (esto es, con una desviación estándar menor) y mediante el mismo método, será de $n X$, ya que deberá utilizarse la media de $n$ resultados independientes. En la práctica, puede resultar preferible cambiar de método para alcanzar la precisión requerida; sin embargo, y en el peor de los casos, no se necesitará gastar más de $n X$. Para poner estos costos dentro de contexto, hay que considerar las pérdidas financieras que le pueden suponer al usuario final de los datos, como resultado del error total que acompaña a los resultados obtenidos. Siempre existen unos valores óptimos de $v_{m}$ y $v_{a}$ para los cuales las pérdidas financieras finales son mínimas.

Otro concepto de gran importancia es el denominado Minimalismo Analítico (2). En el minimalismo analítico, cada etapa del análisis es evaluada al objeto de minimizar los parámetros más significativos, tales como: tiempo, costo, requerimientos de preparación de muestra, consumo de reactivos y de energía y productos de desecho que se originan. Con frecuencia, todos estos parámetros están interrelacionados. Si, por ejemplo, el objetivo de la digestión de la muestra es el de disolución completa, el tiempo de digestión por técnicas convencionales de calefacción puede reducirse considerablemente si se aplica la digestión bajo presión con calentamiento mediante microondas. Por otra parte, el desarrollo de técnicas simples y rápidas para digestiones parciales puede ser de interés y debe ser evaluado. El objetivo del Minimalismo Analítico es llegar a un procedimiento analítico lo más simple posible con un consumo mínimo de recursos. Todo proceso llevado a cabo en un laboratorio, desde la manipulación de la muestra hasta el cálculo de resultados y elaboración del informe, debe considerarse bajo este principio.

Los avances en la instrumentación analítica a lo largo de los últimos 10-15 años han sido espectaculares, permitiendo cada vez la detección de menores contenidos y mejorando la precisión. Pero la preparación previa de la muestra sigue teniendo una importancia primordial. La preparación de la muestra puede resultar frustrante y, a la vez, conducir a conclusiones erróneas si no se le presta la atención debida.

Durante la última década, varios fenómenos han influido en las actividades analíticas:

- El progreso de la técnica en general.

- La automatización de los procesos industriales, que han incrementado la necesidad de medidas más exactas para el control de los mismos, en tiempo real.

- La creciente importancia dada a la protección del medio ambiente, lo cual implica un control analítico fiable.

- El aumento del comercio internacional y la necesidad de reducir las barreras técnicas a dicho comercio, barreras debidas a requerimientos técnicos y a la multiplicidad de procedimientos de evaluación de la conformidad. 


\section{CALIDAD ANALÍTICA}

Sin duda, los usuarios no utilizan un determinado material basándose en su composición química; los materiales se utilizan teniendo en cuenta sus propiedades físicas y mecánicas, buscando una garantía de que el producto sea adecuado para su posterior aplicación; en definitiva, satisfaciendo la demanda del mercado final. Esto no debe interpretarse como un menosprecio al análisis químico, ya que, en realidad, constituye el único medio capaz de proporcionar el control rápido y exacto exigido por los modernos procesos metalúrgicos para garantizar la calidad de sus productos. En definitiva, puede decirse que las actividades analíticas están directamente involucradas en el desarrollo económico mundial. Muchas de las decisiones que se toman en los procesos tecnológicos están basadas en diferentes tipos de ensayos, donde el análisis químico juega un importante papel. Para asegurar decisiones correctas es importante que todas las medidas en las cuales se basan dichos ensayos sean exactas. Un aspecto importante cuando se evalúa la exactitud es la trazabilidad.

Una publicación de Ramsey (3), en la cual se discuten unos resultados obtenidos por ICP-OES, empieza con la frase: "Todo resultado analítico es erróneo, la cuestión es conocer la cuantía de dicho error". Lo importante es poder demostrar la validez de un resultado y determinar los intervalos de confianza; previo a esto, todos los resultados deben ser considerados incorrectos. El significado de la exactitud a veces está mal interpretado. La definición de exactitud según la norma ISO 5725 es: "La proximidad de concordancia entre el resultado del ensayo y el valor de referencia aceptado". Esta definición resulta muy pobre si se compara con la definición metrológica normal, donde el resultado de un ensayo se compara con el resultado verdadero. En la exactitud, o mejor en la inexactitud, hay que considerar dos tipos de error: error sistemático y error casual. El objetivo en todo resultado analítico es que el error sistemático sea cero, aunque en la práctica no siempre ocurre. Con el error casual hay que contar siempre. Consiguientemente, siempre hay un error asociado a todo resultado analítico, por lo cual debe presentarse con una incertidumbre, que es un rango de valores entre los cuales el valor verdadero, o el valor de referencia, se asegura que está con un nivel de confianza determinado. Para una aplicación beneficiosa de las técnicas analíticas, es necesario aplicar criterios claramente definidos para realizar los análisis y para presentar los resultados. De esta manera, se mejora el entendimiento entre ambas partes: entre el responsable analítico y el usuario de los datos, el cual debe tomar decisiones basadas en resultados analíticos fiables. Conseguir los mejores resultados es el objetivo de los respon- sables de todo laboratorio analítico. Esta misión sólo puede conseguirse si dichos responsables tienen la oportunidad de intercambiar sus experiencias con especialistas que trabajen en el mismo campo y que estén al día en los avances instrumentales.

\section{IMPLICACIÓN EN EL CAMPO SIDERÚRGICO}

Aunque las tecnologías analíticas avanzan sin cesar, alcanzándose cada vez mayor rapidez, mejor precisión y exactitud y niveles de detección más bajos, ello no implica que se haya alcanzado la meta buscada, ya que la competitividad industrial hace que las demandas analíticas crezcan como mínimo a la misma velocidad que los mencionados avances. Concretamente en el campo de la industria siderúrgica, se puede ilustrar esta creciente demanda con algunos ejemplos concretos, en los cuales se demuestra claramente la implicación analítica (4).

\subsection{Industria del automóvil}

Las exigencias de los materiales para ser utilizados como chapas para carrocerías las satisfacen aceros con muy bajos contenidos de carbono $(<35$ ppm) y con contenidos de titanio entre 50 y 90 ppm (para retener el nitrógeno libre). Cuando dichos materiales fueron propuestos por primera vez, ninguna de las técnicas en uso: ni la espectrometría de chispa (OES), ni la combustión en horno de inducción con detección por infrarrojo (HFIR), daban una precisión adecuada para la determinación del carbono en estos niveles. Por otra parte, tampoco se disponía de los correspondientes Materiales de Referencia Certificados, para validar los resultados.

Con los desarrollos que han tenido lugar en esta área y con la buena práctica de los laboratorios, la determinación del carbono en estos niveles es actualmente un proceso de rutina que permite determinar contenidos de carbono del orden de $30 \mathrm{ppm}$ con una precisión de $0,6 \mathrm{ppm}$. Como consecuencia, se puede realizar toma de muestras a lo largo del proceso de desgasificación para monitorizar la descarburación y asegurar de esta manera un buen control operacional.

Por otra parte, las chapas de acero galvanizado que se utilizan para posteriormente tratarlas superficialmente y pintarlas, constituye una de las áreas más críticas de la competitividad entre los fabricantes del sector. Si el contenido de hidrógeno del acero, antes del galvanizado, excede el nivel crítico de 0,225 ppm, dicho hidrógeno se sitúa en la interfase cinc-acero dando lugar a porosidades que 
afectan al posterior proceso de pintado y favorecen la corrosión. Al principio de los 90 , la determinación de contenidos de hidrógeno por debajo de 1 ppm no era aún posible. Posteriores desarrollos de equipos con alta sensibilidad permiten actualmente medidas de hidrógeno por debajo de $0,1 \mathrm{ppm}$, dando lugar a la información necesaria para optimizar los parámetros que conducen a la obtención de chapas con contenidos mínimos de hidrógeno, asegurando que todo el material fabricado tenga contenidos por debajo del valor crítico de $0,225 \mathrm{ppm}$ de hidrógeno. El estudio de los perfiles de concentraciones en profundidad de las distintas capas que se originan en un proceso completo de acabado de las chapas para carrocerías, hasta muy recientemente no podía realizarse. El actual desarrollo de las técnicas espectroscópicas con lámpara luminiscente alimentada por radiofrecuencia ha permitido abordar este tema.

Con relación a las piezas mecánicas de la industria de la automoción, conviene recordar que para poder fabricar piezas consistentes, dimensionalmente precisas y a bajo costo, es esencial que el material tenga una buena respuesta a los tratamientos térmicos y que presente una deformación mínima, asegurándose así el correcto comportamiento de las piezas a lo largo de la vida media prevista para el vehículo. La respuesta a los tratamientos térmicos se valora tradicionalmente a través de la medida de dureza mediante el ensayo Jominy. La dureza depende de la composición química y con el fin de obtener una medida más representativa de la respuesta de un acero a los tratamientos térmicos, actualmente se calcula un valor de la dureza basado en el análisis químico (5).

Un rango analítico típico para un material con una dureza estrechamente controlada, con vistas a ser utilizado en piezas de la industria de automoción es: 0,16-0,18 \% C; 0,20-0,35\% Si; 0,85-0,90 $\% \mathrm{Mn},<0,035 \% \mathrm{P} ; 0,020-0,035 \% \mathrm{~S} ; 1,00-1,05 \%$ $\mathrm{Cr} ; 0,22-0,24 \% \mathrm{Mo} ; 0,10-0,20 \% \mathrm{Ni} ;<0,30 \% \mathrm{Cu}$; $0,03-0,04 \% \mathrm{Al} ;<0,015 \% \mathrm{~N}$.

\subsection{Turbinas para la generación de electricidad}

La producción de electricidad está supeditada a la utilización de grandes turbinas, cuyos componentes deben pasar un control de calidad muy estricto. En los últimos 15 años, se ha desarrollado y mejorado notablemente el tipo de material utilizado, a través de una investigación sobre el efecto de los elementos residuales: $\mathrm{P}, \mathrm{As}, \mathrm{Sb}$ y $\mathrm{Cu}$, en la fragilidad y la ductilidad a altas temperaturas. El algoritmo adoptado es:

$$
\begin{gathered}
R(\% \text { factor residual })=P+2,3 \mathrm{As}+3,57 \mathrm{Sn}+ \\
+8,16 \mathrm{Sb}+0,13 \mathrm{Cu}
\end{gathered}
$$

\subsection{Hojalatas para la industria alimentaria}

La hojalata es uno de los productos más importantes de la industria siderúrgica. La ductilidad del acero base es tan importante como lo es en el caso de la industria de la automoción; además, la ausencia de inclusiones es aún más importante. El electroestañado implica una serie de operaciones continuas y el control de todos estos pasos es vital para la obtención de un perfecto producto final. En este campo, la utilización de la espectroscopia de plasma ha desempeñado un papel de gran trascendencia.

\subsection{Control medioambiental}

Las exigencias relativas al control medioambiental están aumentando de una manera muy significativa, lo cual implicará unas crecientes demandas analíticas en el futuro próximo. En este campo, sin duda que la técnica de ICP-MS tiene un papel importante que desempeñar.

\section{TENDENCIAS EN RUTINAS ANALÍTICAS}

Las diferentes técnicas basadas en Espectroscopia Atómica son las más ampliamente difundidas para el análisis elemental de la mayoría de las matrices imaginables, especialmente las metálicas. Fue hacia la mitad de la década de los 40 , cuando se introdujeron los métodos espectroscópicos, con los primeros espectrómetros de emisión. Un avance importante surgió en los años 50 con la introducción de la fluorescencia de rayos X. Seguidamente fueron, la absorción atómica, en la década de los 60, y la espectrometría de plasma, a finales de los 70 , las técnicas innovadoras. Y, más recientemente, en los años 80-90, el desarrollo de la espectrometría de masas con fuente de plasma y de las espectrometrías con lámpara luminiscente han sido las técnicas instrumentales que han venido a dar un avance importante a las tecnologías analíticas.

En cuanto a los laboratorios analíticos, sin duda el cambio más drástico ha tenido lugar hacia la mitad de los años 80, al transformarse los clásicos laboratorios de análisis químico en sofisticados laboratorios fisico-químicos, donde, con sólo presionar un botón, es posible realizar muchos de los trabajos que con anterioridad precisaban mucho tiempo y destreza. Sin duda, el operador o el robot no tienen por qué tener grandes conocimientos químicos, cuando se trata de realizar el control rápido de un proceso; sin embargo, sí se necesita un importante soporte analítico, cuando se trata de realizar una operación analítica de las denominadas de rutina. En la figura 1 se sintetizan las necesidades de formación analítica para el soporte de una rutina 


\section{Soporte para una Rutina Analítica}

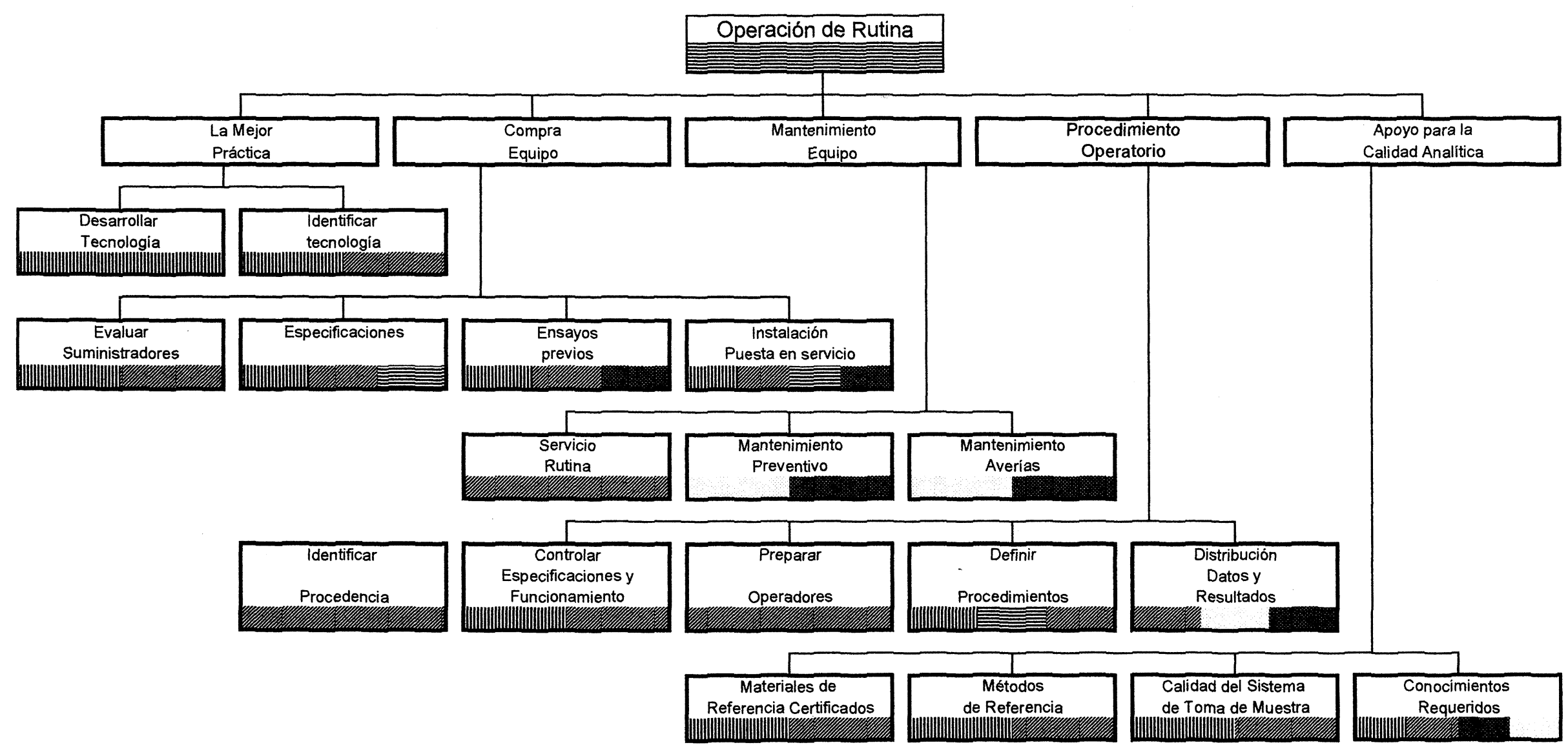

\section{FORMACIÓN}
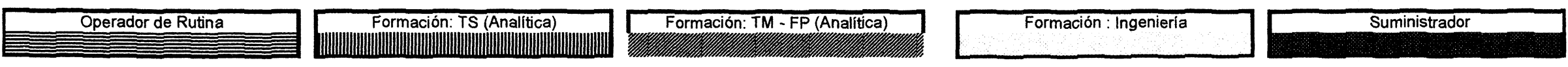

FIG. 1.- Soporte para una rutina analítica

FIG. 1.- Support for a routine analytical facility 
analítica (por cortesía de Mr. Jowitt, British Steel, Teesside Technology Centre).

\section{CONCLUSIÓN}

Tomando como base la experiencia pasada, en el futuro será necesario controlar niveles de elementos residuales cada vez más bajos, con rangos de tolerancias menores y con una trazabilidad garantizada. Simultáneamente, se necesitará mayor rapidez en los resultados y, a poder ser, análisis in situ, ya que este sistema proporciona la forma más directa de controlar un proceso, ofreciendo la información requerida en tiempo real. Otro punto de gran importancia es la posible automatización del conjunto de los procesos analíticos operativos en un determinado laboratorio. No se debe olvidar que, además de la necesidad de controlar los complejos procesos metalúrgicos, existen necesidades análíticas para controlar todos los materiales involucrados en los mismos (materias primas, productos terminados, productos de desecho, etc.). En numerosos casos, estos análisis, a pesar de su carácter rutinario, requieren disponer de una avanzada tecnología y de una sólida formación analítica.

\section{REFERENCIAS}

(1) Thompson, M. y Fearn, T. Analyst, 121, 1996: 275-278.

(2) Halls, D.J. J. Anal. Atom. Spectrom., 10, 1995: 169-175.

(3) Ramsey, M.H., Thompson, M. y Banerjee, E.K. Anal.Proc.,24, 1987: 260-264.

(4) Edington, J.W. y Jowitt, R. Progress in Analytical Chemistry in the Steel and Metals Industry. Ed. R.Nauche. Luxembourg. EC, ISBN 92-827-5706-0, 1996, 8-18.

(5) Geary, E.A., CooK, W.T. y Lane, K.A.G. Heat treatment of Metals, 4, 1993: 95-98. 